Proceedings

\title{
Experimental Tests on TIR Multispectral Images for Temperature-Emissivity Separation by Using the MaxEnTES Algorithm ${ }^{+}$
}

\author{
Vanni Nardino, Gabriele Amato, Donatella Guzzi, Cinzia Lastri and Valentina Raimondi * \\ IFAC-CNR, via Madonna del Piano 10, 50019Sesto Fiorentino, Italy; v.nardino@ifac.cnr.it (V.N.); \\ g.amato@ifac.cnr.it (G.A.); d.guzzi@ifac.cnr.it (D.G.); c.lastri@ifac.cnr.it (C.L.) \\ * Correspondence: v.raimondi@ifac.cnr.it; Tel.: +39-055-5226379 \\ + Presented at the 15th International Workshop on Advanced Infrared Technology and Applications (AITA \\ 2019), Florence, Italy, 17-19 September 2019.
}

Published: 18 September 2019

\begin{abstract}
Satellite images in the TIR are relevant for several Earth Observation applications. The retrieval of temperature and emissivity from the emitted radiance, however, requires the use of suitable algorithms, such as the MaxEnTES that uses the concept of maximum entropy to solve the Temperature-Emissivity Separation problem. Here we discuss the performance of MaxEnTES when applied to TIR images with a limited number of channels, specifically simulated HyspIRI multispectral images and real multispectral images by ASTER. The results were respectively compared with the original temperatures used for the simulations and with the temperatures obtained by using the ASTER TES algorithm.
\end{abstract}

Keywords: temperature-emissivity separation; maximum entropy; thermal infrared; multispectral images; earth observation

\section{Introduction}

Images of the Earth surface, acquired in Thermal Infrared (TIR), provide relevant information for climatological studies, meteorology and surface characterization. Radiance emission in the TIR is modelled by Planck's law that relates the emitted spectral radiance to the target's temperature and emissivity. The retrieval of temperature and emissivity from the emitted radiance-the Temperature/Emissivity Separation (TES) - is an ill-posed problem since the number of available measurements ' $n$ ' is less than the number of unknowns, that are ' $n+1$ '. Several approaches have been proposed to solve the so-called TES problem [1-4]. All these approaches rely on a-priori assumptions to solve the TES problem. Following the work of Jaynes [5], new methods of inverse-modelling for such cases, in which the number of unknowns exceeds the number of measurements, were suggested. These procedures take the collective name of Maximum Entropy formalism (MaxEnt) [5]. Following MaxEnt methodology, the MaxEnTES algorithm retrieves temperature and spectral emissivity as expectation values of the corresponding random variables [6]. Thus, TES is turned into the problem of assessing the Probability Density Function (PDF) of the corresponding random variables that maximize the entropy. The MaxEnt is the less committal approach for bridging the gap between measurements and estimates. MaxEnTES approach, however, requires the availability of several measurements (spectral channels) to provide a good estimate and, in general, its performance improves as the number of measurements increases [6].

In this paper we discuss the performance of MaxEnTES when applied to images featuring a limited number of measurements. Here MaxEnTES was first tested on simulated HyspIRI-like 
multispectral images (7 spectral bands in the TIR) and the results compared with the original ones used for the simulations. Secondly, MaxEnTES was applied to real multispectral images acquired by ASTER ( 5 spectral bands in the TIR) and the results compared with those obtained by using the ASTER-TES algorithm [2].

\section{Materials and Methods}

\subsection{The MaxEnTES Algorithm}

Spectral radiance in the TIR can be expressed as:

$$
L_{i}=\varepsilon\left(\lambda_{i}\right) B\left(T, \lambda_{i}\right),
$$

where $\lambda_{i}$ is the wavelength of the $i$-th channel with $i=1, \ldots, n, B\left(T, \lambda_{i}\right)$ is the Planck function, and $\varepsilon\left(\lambda_{i}\right)$ is the emissivity. The entropy $H$ is an estimate of information content. Given a $P D F=p\left(x_{i}\right)$, the entropy $H$ can be written as:

$$
H=-\sum_{i} p\left(x_{i}\right) \log p\left(x_{i}\right) \quad p\left(x_{i}\right)=\frac{\exp \left(l_{1} f_{1}\left(x_{i}\right)+\ldots+l_{m} f_{m}\left(x_{i}\right)\right)}{Z\left(l_{1}, \ldots, l_{m}\right)}
$$

PDF is expressed as a function of the measurements $f_{i}(x)$, of the partition function $Z\left(l_{1}, \ldots, l_{n}\right)$ and the Lagrange multipliers $\left(l_{1}, \ldots, l_{n}\right)$. Lagrange multipliers result from the constrained maximization of the entropy. The MaxEnt approach consists in finding the optimal value of Lagrange multipliers ( $l_{1}$, $\left.\ldots, l_{n}\right)$ that matches the expectations $E\left[f_{i}(x)\right]$ to the available measurements $L_{i}$.

The MaxEnt method applied to TES problem can be summarized with the following statement: "finding the PDF with maximum information entropy that maximizes the probability to obtain the available experimental results $L_{i}$ when measuring $f_{i}(x)^{\prime \prime}$. The goal is the estimation of both temperature $T$ and emissivity spectrum $\varepsilon \mathrm{I}$, calculated in the generic $i$-th channels, as expectation values of the corresponding aleatory variables $e_{i}, t$. Setting temperature and emissivity as $T=E\{t\}, \varepsilon_{k}$ $=E\left\{e_{k}\right\}$ : the spectral radiance $L i$ can be retrieved as:

$$
f_{i}(\vec{e}, t)=e_{i} B\left(t, \lambda_{i}\right) \quad ; \quad L_{i}=E\left\{f_{i}(x)\right\}=\int_{\Re} p(x) f_{i}(x) d x \quad ; \quad \forall i
$$

\subsection{Simulated Data}

The simulated data used for testing the MaxEnTES algorithm were generated according to the parameters of an acquisition performed by the HyspIRI TIR instrument [7]. The instrument has a spatial sampling of $60 \mathrm{~m}$ and acquires images in 7 spectral bands between 7 and $12 \mu \mathrm{m}$, as reported in Table 1. The HyspIRI-like image was simulated selecting 19 different classes of targets from the ECOSTRESS spectral library [8,9] Figure 1. The selected classes were used for generating a HyspIRIlike image of surface emitted radiance, that was used as an input for the MaxEnTES algorithm. White noise was considered in the simulation.

Table 1. HyspIRI TIR spectral bands.

\begin{tabular}{ccc}
\hline Band & $\lambda(\mu \mathrm{m})$ & Bandwidth $(\mu \mathrm{m})$ \\
\hline Band 1 & 7.35 & 0.320 \\
Band 2 & 8.28 & 0.340 \\
Band 3 & 8.63 & 0.350 \\
Band 4 & 9.07 & 0.360 \\
Band 5 & 10.53 & 0.540 \\
Band 6 & 11.33 & 0.540 \\
Band 7 & 12.05 & 0.520 \\
\hline
\end{tabular}




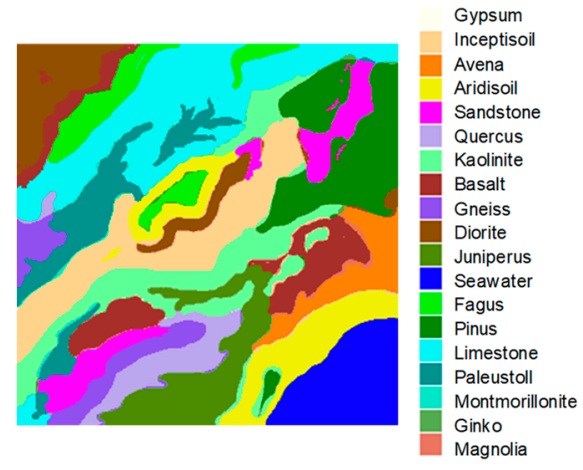

(a)

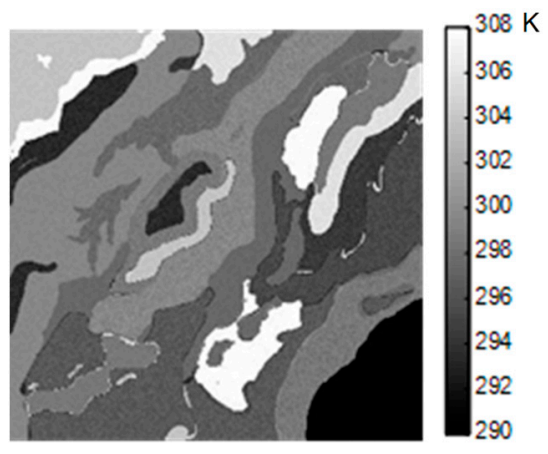

(b)

Figure 1. HyspIRI-like simulated image: (a) thematic map with 19 classes; (b) Simulated ground-truth temperature image.

\subsection{Real Satellite Data}

The ASTER surface radiance (A09 product) image used for testing the MaxEnTES was acquired over the Fucino plains (Abruzzo, Italy) on 8 August 2018 at 10:55 local time (Figure 2a). ASTER is a multispectral imager with 5 spectral bands in the $8 \mu \mathrm{m}-11 \mu \mathrm{m}$ spectral range, as shown in Table 2 . Figure $2 \mathrm{~b}$ shows the temperature image as provided by ASTER A08 product.

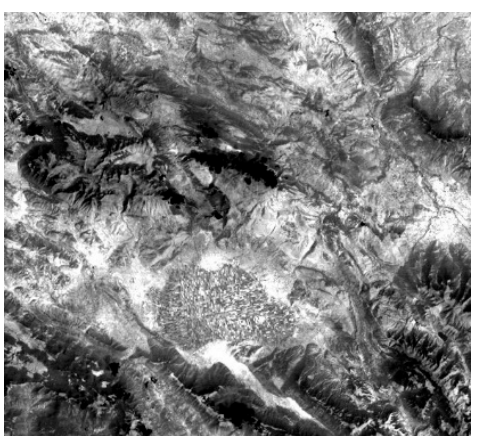

(a)

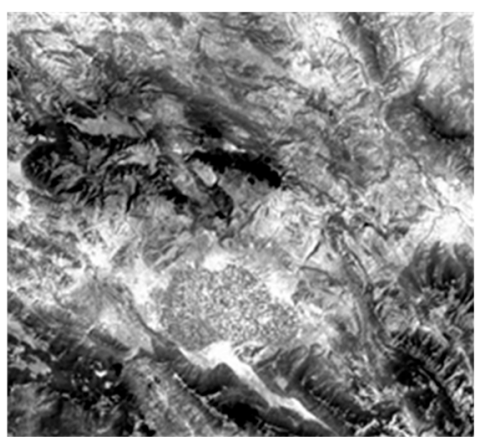

(b)

Figure 2. ASTER acquisition over Fucino area, Abruzzo, Italy: (a) ASTER A09 product-Ground emitted radiance image at $10.657 \mu \mathrm{m}$, (b) ASTER A08 product-Temperature image.

Table 2. ASTER TIR spectral bands.

\begin{tabular}{ccc}
\hline Band & $\lambda \min (\mu \mathrm{m})$ & $\lambda \max (\mu \mathrm{m})$ \\
\hline Band 10 & 8.12 & 8.47 \\
Band 11 & 8.47 & 8.82 \\
Band 12 & 8.92 & 9.27 \\
Band 13 & 10.25 & 10.95 \\
Band 14 & 10.95 & 11.65 \\
\hline
\end{tabular}

\section{Results}

The MaxEnTES algorithm was applied to the HyspIRI-like simulated data in order to retrieve the temperature image (not shown) and the retrieved temperature values were compared with those used for the simulation. The histogram of their differences is plotted in Figure 3a. The errorestimated as Full Width Half Maximum - in retrieved temperature $\Delta \mathrm{T}$ is equal to $\pm 1.5 \mathrm{~K}$.

As far as real satellite data are concerned, the differences between MaxEnTES and ASTER-TES retrieved temperatures exhibit a $\Delta \mathrm{T} \approx \pm 2.5 \mathrm{~K}$ at FWHM. Figure $3 \mathrm{~b}$ shows the scatter plot between temperatures retrieved by using MaxEnTES and ASTER-TES, respectively. $\mathrm{R}^{2}$ is equal to 0.78 . 


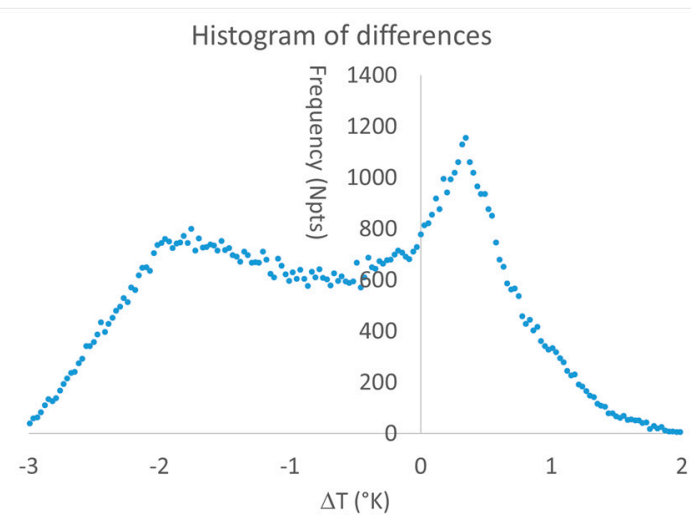

(a)

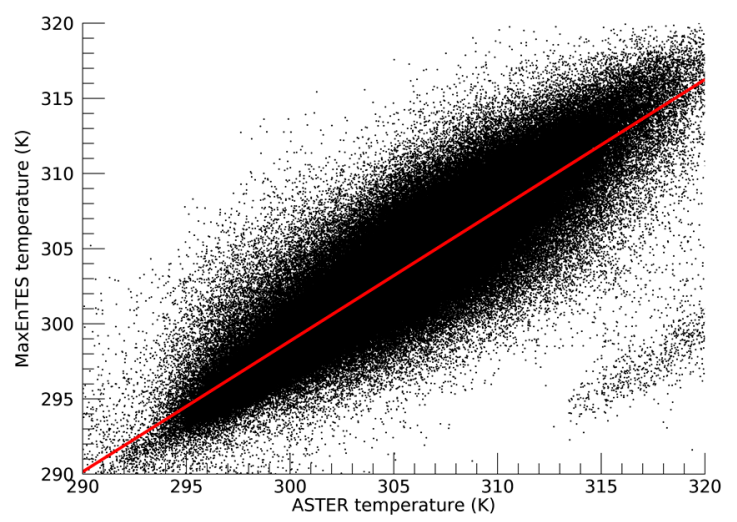

(b)

Figure 3. MaxEnTES applied to simulated and real data: (a) Simulated data: histogram of the differences between the temperatures retrieved using MaxEnTES and those used for the simulation. (b) Real data: scatterplot of ASTER A08 temperatures vs the temperatures retrieved using MaxEnTES.

\section{Discussion and Conclusions}

Although MaxEnTES provides an optimal performance when applied to hyperspectral images that feature a large number of channels ( $>10$ channels) as already demonstrated in [4], the results obtained in this paper showed good performance of the algorithm even when applied to multispectral images in the TIR, at least when the number of channels of the image is close to 10 . The performance, in fact, apparently deteriorates when applied to the ASTER data featuring only 5 channels, although the results are still consistent with those obtained using the standard ASTER-TES algorithm. Next actions of the research aim to apply MaxEnTES to real multispectral images in the MIR-TIR featuring a larger number of channels.

Author Contributions: Conceptualization, C.L., D.G. and V.R.; data simulation, C.L.; data acquisition, D.G., G.A.; data processing, C.L., D.G. and V.N.; data analysis, D.G., C.L. and V.R.; writing, D.G. and V.R.

Funding: This research was partly funded by OT4CLIMA, financed by Italian Ministry of Research, and by ARCO-IPERCONTROL, funded by Tuscany Region with a research grant that is $50 \%$-financed with the resources of the ESF 2014-2020 ROP in the frame of Giovanisì programme (www.giovanisi.it) for the autonomy of young people.

Conflicts of Interest: The authors declare no conflict of interest.

\section{References}

1. Barducci, A.; Pippi, I. Temperature and emissivity retrieval from remotely sensed images using the "Grey body emissivity" method. IEEE Trans. Geosci. Remote. Sens. 1996, 34, 681-695.

2. Gillespie, A.; Rokugawa, S.; Matsunaga, T.; Cothern, J.; Hook, S.; Kahle, A. A temperature and emissivity separation algorithm for Advanced Spaceborne Thermal Emission and Reflection Radiometer (ASTER) images. IEEE Trans. Geosci. Remote. Sens. 1998, 36, 1113-1126.

3. Kahle, A.B.; Madura, D.P.; Soha, J.M. Middle infrared multispectral aircraft scanner data: Analysis for geological applications. Appl. Opt. 1980, 19, 2279.

4. Palluconi, F.; Kahle, A.; Gillespie, A.; Gu, D. Autonomous atmospheric compensation (AAC) of high resolution hyperspectral thermal infrared remote-sensing imagery. IEEE Trans. Geosci. Remote. Sens. 2000, $38,2557-2570$.

5. Jaynes, E.T. Information Theory and Statistical Mechanics. Phys. Rev. 1957, 106, 620-630.

6. Barducci, A.; Guzzi, D.; Lastri, C.; Marcoionni, P.; Nardino, V.; Pippi, I. Maximum Entropy TemperatureEmissivity Separation in the TIR spectral range using the MaxEnTES algorithm. Infrared Phys. Technol. 2013, $56,12-20$.

7. Lee, C.M.; Cable, M.L.; Hook, S.J.; Green, R.O.; Ustin, S.L.; Mandl, D.J.; Middleton, E.M. An introduction to the NASA Hyperspectral InfraRed Imager (HyspIRI) mission and preparatory activities. Remote. Sens. Environ. 2015, 167, 6-19. 
8. Meerdink, S.K.; Hook, S.J.; Roberts, D.A.; Abbott, E.A. The ECOSTRESS spectral library version 1.0. Remote. Sens. Environ. 2019, 230, 111196.

9. Baldridge, A.; Hook, S.; Grove, C.; Rivera, G. The ASTER spectral library version 2.0. Remote. Sens. Environ. 2009, 113, 711-715.

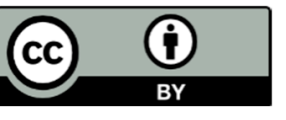

(C) 2019 by the authors. Licensee MDPI, Basel, Switzerland. This article is an open access article distributed under the terms and conditions of the Creative Commons Attribution (CC BY) license (http://creativecommons.org/licenses/by/4.0/). 